\title{
Téoros
}

Revue de recherche en tourisme

\section{La construction des connaissances en tourisme à l'université} La production de mémoires et thèses liés au tourisme à l'UQAM (1978 à 2005)

\section{Boualem Kadri et François Bédard}

Volume 25, numéro 2, été 2006

URI : https://id.erudit.org/iderudit/1071071ar

DOI : https://doi.org/10.7202/1071071ar

Aller au sommaire du numéro

Éditeur(s)

Université du Québec à Montréal

ISSN

0712-8657 (imprimé)

1923-2705 (numérique)

Découvrir la revue

Citer cette note

Kadri, B. \& Bédard, F. (2006). La construction des connaissances en tourisme à l'université : la production de mémoires et thèses liés au tourisme à l'UQAM (1978 à 2005). Téoros, 25(2), 78-80. https://doi.org/10.7202/1071071ar 


\section{La construction des connaissances en tourisme à l'université \\ La production de mémoires et thèses liés au tourisme à I'UQAM (1978 à 2005)}

\section{Boualem Kadri et François Bédard}

La production des connaissances en milieu universitaire par les thèses est une forme de la construction du savoir dont l'analyse attire peu l'intérêt des chercheurs. Peu d'études, semble-t-il, sont en effet consacrées à cette face cachée du processus de la construction des connaissances en tourisme. À titre d'exemple, on relèvera dans la Revue Annals of Tourism Research, aux USA, seulement trois analyses consacrées aux thèses de doctorat produites de 1971 à $1998^{1}$. Pourtant, cette forme de la production scientifique en milieu universitaire peut révéler les forces et les faiblesses inhérentes au processus de construction du savoir dans un champ de connaissances donné, en l'occurrence celui du tourisme. Le présent texte vise à apporter une contribution à cet égard et, pour ce faire, nous avons choisi le cas de l'Université du Québec à Montréal (UQAM).

\section{Méthodologie}

Compte tenu de la faible production de thèses de doctorat liées à la dimension touristique à I'UQAM, nous avons ajouté à notre recherche la réalisation des mémoires de maîtrise. La base de données de l'UQAM répertorie les travaux de maîtrise et de doctorat sous le terme de " thèses " ; nous utiliserons ce terme pour identifier la production (maîtrise et doctorat), mais nous distinguerons dans notre analyse le mémoire de maîtrise et la thèse de doctorat.

Pour cette recherche, nous avons considéré la période 1978-2005, laquelle couvre près de trois décennies. Le recensement de la production scientifique a été réalisé à partir de la base de données fournie par le Service de recherche documentaire de I'UQAM et disponible en ligne ${ }^{2}$. Nous avons utilisé les mots-clés tourisme-loisir-voyage/voyageur pour ce recensement, ce qui nous a permis de saisir la diversité disciplinaire observée dans la production des thèses liées au tourisme à l'UQAM. Le mot « tourisme » dans notre texte inclut les dimensions de loisir et de voyage.

Nous avons recensé, depuis la réalisation de la première thèse (maîtrise) liée au tourisme, en 1978, 65 mémoires de maîtrise et thèses de doctorat produits dans le cadre de divers programmes disciplinaires de deuxième et de troisième cycles de I'UQAM. Nous avons ajouté à ce relevé 5 thèses de doctorat réalisées dans des universités à l'étranger par des personnes rattachées au Département d'études urbaines et touristiques de l'École des sciences de la gestion de l'UQAM.
Le nombre total de thèses utilisé pour notre analyse est donc de 70, c'est-à-dire 59 maîtrises et 11 doctorats.

\section{Un intérêt disciplinaire diversifié pour le tourisme}

Le tableau 1 permet d'observer qu'une diversité de disciplines (administration des affaires, géographie, environnement, politique, droit, études littéraires, etc.) participent à la production de mémoires de mâ̂trise et de thèses de doctorat en tourisme; nous en avons ainsi relevé une quinzaine, associées à divers programmes de deuxième et de troisième cycles. Notons cependant que leur contribution à la recherche en tourisme apparait inégale, bien que deux d'entre elles dominent ce portrait : administration des affaires (13 maîtrises, 2 doctorats) et géographie (14 maîtrises), représentant respectivement $21,4 \%$ et $20 \%$ et totalisant à elles seules $41,4 \%$ des thèses produites au cours de la période 1978-2005. Ces deux disciplines ont une part presque équivalente dans cette production, avec toutefois une légère avancée de la première en matière de réalisation doctorale.

D'autres disciplines se distinguent également dans ce portrait général, mais avec des contributions différentes et moindres que celles réalisées par les deux disciplines dominantes identifiées précédemment:

- communication : 8 mémoires de maîtrise, soit 11,4 \%,

- sciences de l'environnement : 6 mémoires de maîtrise, soit 8,6 \%,

- études littéraires : 5 mémoires de maîtrise, soit 7,1%,

- science politique : 2 mémoires de maîtrise et 2 thèses de doctorat (dont 1 réalisée à l'étranger), soit près de $6 \%$ du total de la production.

Ce premier résultat met en évidence, d'une part, l'empreinte de la géographie encore vivace sur les plans théorique et méthodologique dans les problématiques du développement touristique et, d'autre part, l'intérêt d'une analyse du tourisme sous l'angle de la gestion, favorisée par une culture managériale bien ancrée en Amérique du Nord. Par ailleurs, nous relevons l'importance des disciplines attachées aux sciences humaines, notamment (tableau 2), et à celles des sciences de la gestion, structurant toutes deux le portrait de la recherche universitaire en tourisme par les thèses. En effet, les sciences humaines totalisent une production de 27 thèses (24 maîtrises, 3 doctorats), représentant $41,5 \%$ du total de la production, alors que les sciences de la gestion totalisent 21 thèses (18 maîtrises, 3 doctorats), représentant 32 \% du total des thèses réalisées à l'UQAM. 


\section{Tableau 1}

Portrait général de la production de mémoires de maîtrise et de thèses de doctorat en tourisme à l'UQAM, selon les programmes disciplinaires (1978-2005)

\begin{tabular}{|c|c|c|c|c|c|c|c|c|c|}
\hline \multirow[t]{3}{*}{ Discipline } & \multicolumn{6}{|c|}{ Période } & & & \\
\hline & \multicolumn{2}{|c|}{$1978-1987$} & \multicolumn{2}{|c|}{$1988-1997$} & \multicolumn{2}{|c|}{ 1998-2005 } & \multicolumn{2}{|c|}{ Total } & \multirow{2}{*}{$\begin{array}{l}\text { Total } \\
M+D\end{array}$} \\
\hline & M & D & M & D & M & D & M & D & \\
\hline $\begin{array}{l}\text { Administration } \\
\text { des affaires }\end{array}$ & - & - & 4 & - & 9 & $2\left(1^{\star}\right)$ & 13 & 2 & 15 \\
\hline Géographie & 3 & - & 7 & - & 4 & - & 14 & - & 14 \\
\hline Communication & - & - & 5 & - & 3 & - & 8 & - & 8 \\
\hline $\begin{array}{l}\text { Sciences de } \\
\text { l'environnement }\end{array}$ & - & - & 2 & - & 4 & - & 6 & - & 6 \\
\hline Études littéraires & 1 & - & 2 & - & 2 & - & 5 & - & 5 \\
\hline Science politique & - & - & 2 & - & 1 & $1^{*}$ & 3 & 1 & 4 \\
\hline Sociologie & 1 & - & 1 & - & - & 1 & 2 & 1 & 3 \\
\hline Gestion de projet & - & - & 2 & - & 1 & - & 3 & - & 3 \\
\hline Psychologie & - & - & 1 & - & 1 & 1 & 2 & 1 & 3 \\
\hline Économie & - & $1^{*}$ & - & $1^{*}$ & 1 & - & 1 & 2 & 3 \\
\hline Études urbaines & - & - & - & - & - & 2 & - & 2 & 2 \\
\hline $\begin{array}{l}\text { Informatique } \\
\text { de gestion }\end{array}$ & - & - & - & - & 1 & - & 1 & - & 1 \\
\hline Droit & - & - & - & $1^{*}$ & - & - & - & - & 1 \\
\hline Sémiologie & - & - & - & - & - & 1 & - & 1 & 1 \\
\hline Histoire & - & - & - & - & 1 & - & 1 & - & 1 \\
\hline TOTAL M/D & 5 & 1 & 26 & 2 & 28 & 8 & 59 & 11 & 70 \\
\hline TOTAL M+D & \multicolumn{2}{|c|}{6} & \multicolumn{2}{|c|}{28} & \multicolumn{2}{|c|}{36} & \multicolumn{2}{|c|}{70} & \\
\hline$\%$ & \multicolumn{2}{|c|}{$8,6 \%$} & \multicolumn{2}{|c|}{$40 \%$} & \multicolumn{2}{|c|}{$51,4 \%$} & \multicolumn{2}{|c|}{$100 \%$} & \\
\hline Légende & & & & गсto & 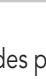 & sonnes r & & & QAM \\
\hline
\end{tabular}

Tableau 2

La production des mémoires de maîtrise et des thèses de doctorat selon les Facultés et École à I'UQAM

\begin{tabular}{l|c|c}
\hline Faculté et École & Maîtrise & Doctorat \\
\hline $\begin{array}{l}\text { Faculté des sciences humaines } \\
\text { (géographie, linguistique et didactique des langues, }\end{array}$ & 24 & 3 \\
$\begin{array}{l}\text { psychologie, sociologie, études littéraires, histoire) } \\
\text { École des sciences de la gestion } \\
\text { (management et technologie, sciences économiques, } \\
\text { stratégie des affaires, études urbaines et touristiques) }\end{array}$ & 18 & 3 \\
$\begin{array}{l}\text { Faculté de communication } \\
\text { (communication sociale et publique) }\end{array}$ & 8 & - \\
$\begin{array}{l}\text { Faculté des sciences } \\
\text { (sciences de l'environnement) }\end{array}$ & 6 & - \\
$\begin{array}{l}\text { Faculté de science politique et droit } \\
\text { (science politique) }\end{array}$ & 3 & - \\
\hline TOTAL M/D & $\mathbf{5 9}$ & $\mathbf{6}$ \\
\hline TOTAL M+D & \multicolumn{6}{|c|}{$\mathbf{6 5}$} & \\
\hline
\end{tabular}

Note : les thèses de doctorat réalisées à l'étranger (soit 5) ne sont pas comprises dans ce tableau.

\section{Une construction des connaissances dominée par les mémoires de maîtrise et réalisée dans d'autres disciplines que le tourisme}

Nous avons observé l'évolution de la production des thèses (maîtrise et doctorat) liées au tourisme sur une période de près de trois décennies. Nous remarquons que la part des mémoires de maîtrise est de $84 \%$, dominant largement cette production scientifique, alors que celle liée aux thèses de doctorat n'est que de $16 \%$, en tenant compte des quelques thèses réalisées à l'étranger par des enseignants de I'UQAM. Cette évolution de la production des connaissances en tourisme-loisir-voyage se fait selon certains contextes au cours des trois périodes.

\section{8-1987 : lente émergence de la reconnaissance du tourisme par les thèses}

Durant cette période décennale, 5 mémoires de maîtrise et 1 thèse de doctorat ont été réalisés, représentant environ 8,6\% de l'ensemble des thèses produites (tableau 1). II s'agit d'une période de démarrage qui a débuté à l'UQAM en 1978, selon les données recueillies. Cette production des connaissances est assurée par la géographie et la sociologie, deux disciplines déjà très impliquées dans l'analyse du phénomène touristique qui en est à ses début en matière de reconnaissance scientifique à l'UQAM. Nous avons relevé à ce propos quelques percées, dix années après la création de I'UQAM en 1968 :

- la mise en place du département d'études urbaines et touristiques
en 1976 ;

- la création du baccalauréat en gestion du tourisme et de l'hôtellerie en 1978 ;

- le lancement de la revue de recherche en tourisme Téoros par un groupe de professeurs du département d'études urbaines et touristiques en 1982.

\section{8-1997 : production et compétition interdisciplinaire}

Les mémoires de maîtrise et les thèses de doctorat recensés durant cette période représentent $40 \%$ de l'ensemble de la production en près de 30 années, à l'UQAM. Nous observons un accroissement de 366 \% par rapport à la période 1978-1987. Si cette production reste dominée par les mémoires de maîtrise (26 maîtrises), nous remarquons aussi l'émergence d'autres disciplines que celles traditionnellement engagées dans la recherche en tourisme, par exemple : sciences de l'environnement (2), communication (5), administration des affaires (4).

Par ailleurs, la production de thèses de doctorat, qui reste très timide, est réalisée en partie dans des universités à l'étranger par un personnel enseignant de I'UQAM.

Néanmoins, la reconnaissance scientifique du tourisme se renforce un peu plus durant cette période en termes de structures et de programme universitaire, notamment par : 
- la mise en place du CIFORT (Centre international de formation et de recherche tourisme, en 1991;

- la création de la Chaire de tourisme, en 1992, qui fait le lien entre l'Université et l'industrie touristique;

- la mise en place du premier programme de deuxième cycle au Québec, celui de la maîtrise en planification et gestion du tourisme, en 1997, programme orienté vers un profil professionnel.

\section{8-2005 : maintien de la production des mémoires de maîtrise et tendance à la croissance pour les thèses de doctorat}

Durant cette période, la production scientifique est de 28 mémoires de maîtrise et de 8 thèses de doctorat, représentant près de $51,4 \%$ de la production réalisée sur près de trois décennies. II s'agit d'un accroissement de 28,6 \% par rapport à la période 1988-1997.

Si le nombre de mémoires de maîtrise (28) réalisés au cours de cette période est presque équivalent à celui de la période précédente (26), c'est toutefois l'augmentation sensible du nombre de doctorats qui va relever la production pour la période 1998-2005 : 8 doctorats contre 2 pendant la décennie précédente.

L'observation de la contribution disciplinaire au tourisme fait ressortir ici deux aspects :

- la discipline administration des affaires domine la production de mémoires de maîtrise (9) et de thèses de doctorat (2), représentant plus de 30,5\% de la production de la période 1998-2005, contre $11 \%$ en géographie ;

- la progression des mémoires de maîtrise en sciences de l'environnement reste encore constante. Cette ouverture aux dimensions environnementale et de développement durable en tourisme, depuis les années 1990, se situe dans un contexte stimulant sur le plan international (sommets de Rio en 1992 et de Johannesburg en 2002), mais surtout sur le plan universitaire, où l'UQAM affiche sa participation dans des projets de partenariat interuniversitare visant le développement durable en Amazonie.

\section{Conclusion}

Ce premier portrait de la recherche en tourisme à l'UQAM par la production des mémoires de maîtrise et des thèses de doctorat dans les programmes de deuxième et de troisième cycles présente, d'une manière globale, deux aspects : une contribution disciplinaire très diversifiée (15 disciplines) et un apport à la formation dominé par les mémoires de maîtrise. Cette situation nous amène à soulever deux problèmes associés à la construction des connaissances en tourisme et à la reconnaissance scientifique de cette discipline :

- Une domination des disciplines autres que celle du tourisme, en matière de production des connaissances, mettant en évidence une faiblesse importante, à savoir l'absence d'une maîtrise en tourisme avec un profil recherche.

- Une faiblesse dans la production de thèses de doctorat s'intéressant aux thématiques touristiques, par rapport aux programmes de maîtrise de diverses disciplines (tableaux 1 et 2). En outre, $45 \%$ des thèses de doctorat ayant une dimension tourisme (5 sur 11) ont été produites dans des universités à l'étranger.

Cette double contrainte impose une sorte de diktat épistémologique et méthodologique au tourisme (concepts, théories et méthodes venant des autres disciplines) et, par là même, freine sa reconnaissance scientifique et son autonomie disciplinaire.

Boualem Kadri est professeur associé au Département d'études urbaines et touristiques

François Bédard est directeur du CIFORT et professeur au même département.

\section{Notes}

1 II s'agit de : Jafari, Jafar, et Dean Aaser (1988), «Tourism as the Subject of Doctoral Dissertations ", Annals of Tourism Research, vol. 15, p. 407-429, University of Winconsin-Stout, USA; Hall, C. Michael (1991), "Tourism as the Subject of Post-graduate Dissertations in Australia ", Annals of Tourism Research, vol. 11, p. 619-621, USA; Pizam, Abraham, et Elanjikal M. Chacko (1982), «Tourism and Hospitality Related Dissertations, 1976-1980", Annals of Tourism Research, vol. 9, p. 587-620, University of Winconsin-Stout, USA.

2 Site de recherche Manitou de la Bibliothèque de I'UQAM, [http:// www.manitou.uqam.ca/manitou.dll]. 